\title{
Aiding Traffic Prediction Servers through Self-Localization to Increase Stability in Complex Vehicular Clustering
}

\author{
Iftikhar Ahmad $\left(\mathbb{D},{ }^{1,2}\right.$ Rafidah Md Noor $\left(\mathbb{D},{ }^{2}\right.$ Roobaea Alroobaea $(D),{ }^{3}$ Muhammad Talha $\left(\mathbb{D},{ }^{4}\right.$ \\ Zaheed Ahmed, ${ }^{5}$ Umm-e- Habiba, ${ }^{5}$ and Ihsan Ali $\mathbb{D}^{2}$ \\ ${ }^{1}$ Department of CS and IT, Mirpur University of Science and Technology (MUST), Mirpur-10250, \\ Azad Jammu and Kashmir (AJK), Pakistan \\ ${ }^{2}$ Department of Computer System and Technology, Faculty of Computer Science and Information Technology, \\ University of Malaya, Kuala Lumpur 50603, Malaysia \\ ${ }^{3}$ Department of Computer Science, College of Computers and Information Technology, Taif University, P. O. Box 11099, \\ Taif 21944, Saudi Arabia \\ ${ }^{4}$ Deanship of Scientific Research, King Saud University, Riyadh, Saudi Arabia \\ ${ }^{5}$ Faculty of Computing \& Engineering, University of Kotli, Azad Jammu and Kashmir (AJK), Pakistan
}

Correspondence should be addressed to Iftikhar Ahmad; ify_ia@yahoo.com and Ihsan Ali; ihsanalichd@siswa.um.edu.my

Received 29 November 2020; Revised 31 December 2020; Accepted 4 January 2021; Published 19 January 2021

Academic Editor: M. Irfan Uddin

Copyright (c) 2021 Iftikhar Ahmad et al. This is an open access article distributed under the Creative Commons Attribution License, which permits unrestricted use, distribution, and reproduction in any medium, provided the original work is properly cited.

\begin{abstract}
The integration of cellular networks and vehicular networks is complex and heterogeneous. Synchronization among vehicles in heterogeneous vehicular clusters plays an important role in effective data sharing and the stability of the cluster. This synchronization depends on the smooth exchange of information between vehicles and remote servers over the Internet. The remote servers predict road traffic patterns by adopting deep learning methods to help drivers on the roads. At the same time, local data processing at the vehicular cluster level may increase the capabilities of remote servers. However, global positioning system (GPS) signal interruption, especially in the urban environment, plays a big part in the detritions of synchronization among the vehicles that lead to the instability of the cluster. Instability of connections is a major hurdle in developing cost-effective solutions for deriving assistance and route planning applications. To solve this problem, a self-localization scheme within the vehicular cluster is proposed. The proposed self-localization scheme handles GPS signal interruption to the vehicle within the cluster. A unique clustering criterion and a synchronization mechanism for sharing traffic information system (TIS) data among multiple vehicles are developed. The developed scheme is simulated and compared with existing known approaches. The results show the better performance of our proposed scheme over others.
\end{abstract}

\section{Introduction}

Smart traffic is vital for economic growth and can only be achieved by reducing the life risk on the road, fuel consumption, environmental impact, and driving time to a minimum. The increasing number of vehicles on the road needs to be handled with emerging information communication technologies. In VANET, traditional constraints always cause link breakage that has a negative impact on the performance of the network. Instability factors should be taken care of, to share data at the right time and place for effective road traffic management. When it comes to heterogeneous networks, stable connection to $3 \mathrm{G} / \mathrm{LTE}$ network is essential for the proper functioning of TMSs [1]. A concept of artificial intelligence such as representation learning has also been used recently for vehicular networks to process vehicular trajectory data and to predict optimal routes [2]. A variety of methods [3] have been proposed to make the cluster stable. However, each method has its pros and cons regarding our TIS scenario $[4,5]$.

Road Traffic Efficiency is the steady and smooth flow of vehicles without any traffic jams on the road. The application was developed to control vehicle flow behavior on the road to 
minimize travel time and reduce/avoid congestion, and such applications are called traffic efficiency applications. These applications are based on Internet services and require quality of service communication for delivery of information on time and at the required location. The quality degrades with the increasing number of cars due to the increase in message exchange, and the example of such applications is floating car data (FCD) services and extended FCD services. This kind of application requires the periodic transmission of vehicle's data collected by road embedded sensors, radars, and cameras to Internet-based remote servers (also known as traffic information servers) [6]. The server processes traffic data, analyzes and predicts traffic congestion, and propagates to the vehicle to make the right decision regarding route planning. In addition to this, the vehicle may request related information concerning their route and driving context periodically and be transferred to the occurrence of an event. There are some related examples of today's applications, such as Waze and Google Maps that can work with on-board units (OBUs) to help assist drivers on the roads.

IoVs rely on smart vehicles that process traffic-related information locally and reduce dependency on a remote server over the Internet. The clustering of vehicles is a good example where a group of vehicles works cooperatively to process data for better road traffic management. The vehicular cluster should be stable enough to act positively to extend the capabilities of IoVs. Driving assistance and route planning applications rely on the reception of GPS signals by the vehicle at the right time and place. In case of interruption of GPS signals, the vehicles are unable to get their location information failing to propagate location information within the cluster. Due to this, the cluster head $(\mathrm{CH})$ is unable to keep a record of the cluster members (CMs) and may opt to remove the vehicle from the CM list once not receiving proper information from $\mathrm{CM}$. This phenomenon increases the instability of the cluster, which leads to performance degradation. The situation may become worse if several vehicles within a cluster are not able to calculate their location coordinates accurately, making it unmanageable for the $\mathrm{CH}$ to control the cluster.

To solve the above-discussed problem, a self-localization vehicular clustering scheme is proposed that encounters GPS interruption in cars moving on the road. First, a heterogeneous vehicular clustering mechanism is developed upon unique criteria. Furthermore, a mathematical formulation is introduced within the clustering process to calculate the location of the typical vehicle that is not receiving any GPS signal. The calculated location information is propagated back to $\mathrm{CH}$ on time that helps in making a collective decision regarding route planning. After the incorporation of the proposed scheme, we found that the synchronization within cluster increased, and so does the stability. The results of the simulation study show that our scheme increases the synchronization of the vehicles among each other, which makes the cluster stable. We compared results with existing schemes such as the Vehicular Multihop algorithm for Stable Clustering (VMaSC) [7] and Mobile Gateway Selection Algorithm (MGSA) [8].

The OpenStreetMap is integrated within the OMNet++ network simulator [9] and Simulation of Urban Mobility
(SUMO) [10]. The rest of the paper is organized into five sections. Section 2 discusses the state-of-the-art literature, and the proposed scheme is presented in Section 3. The simulation study and the discussion on results are presented in Section 4. The conclusion of the paper is presented in Section 5. There is a lack of empirical research within the academic field of IT security that tries to measure the amount of human influence [3]. Some existing empirical studies analyze user perception, behavior, and attitude towards computer ethics and information security [3-5], as computer security and computer ethics are important components of the management information system $[6,11]$.

The rest of the paper is organized into five sections. Related work is discussed in Section 2, and the methodology of the self-localization scheme is discussed in Section 3. Section 4 presents the implementation and comparison of results. The paper is concluded in Section 5.

\section{Related Work}

Broadly two types of vehicular clustering solutions are there denoted as safety and nonsafety. Infotainment applications are the third category; each of these types has its data rate and frequency requirements $[12,13]$. Clustering solutions developed for content downloading [14] are less time-sensitive, but TIS cannot tolerate a delay of over half a second. Similarly, clustering solutions for road safety require considerable prompt information, but no cooperation mechanism is an essential requirement in TIS. A stable cluster, which is developed based on some specific clustering criteria, is required for driving assistance applications. Although some solutions exist $[7,8,15,16]$, they ignore synchronization within cluster that is essential for cluster stability.

The literature related to road traffic efficiency (driving assistance and route planning applications) that incorporates heterogeneous V2-LTE infrastructure is still in its infancy. The work in [7] is mainly developed for road safety and ignores the stability of the cluster and the mechanism of cooperation. The work in [15] presents server-assisted cooperation management that is related to infotainment contents and fails to address instability, which is a major concern for network performance. The work in [8] is developed for $3 \mathrm{G}$ network integration with VANET and neglects cost, stability, and cooperation among CMs.

Here, one thing is worthwhile to mention, TIS works and can perform well only if they are enabled to access the remote server over the Internet and can get information at the right time and place. To access remote servers while moving on the road, vehicles must remain connected to the Internet via thirdparty networks or telecommunication networks such as $3 \mathrm{G}$ or LTE. The use of telecommunication networks for Internet access is not free, and the required data rate and latency must not be greater than the threshold value (as defined in [17]).

In addition to the discussed literature, the schemes that are related to the location calculation of the vehicles for various purposes are focused in our work. Most vehicles in VANET calculate their location or position with the help of GPS by finding out their coordinates. Usually, each vehicle propagates location to each other to take a cooperative 
decision regarding road safety and traffic efficiency that is a built-in feature of dedicated short-range communication. There are TMSs that utilize and make use of the other vehicle's position to make a better decision regarding route planning and driving assistance [18].

One of the popular clustering schemes is named as Vehicular Multihop algorithm for Stable Clustering (VMaSC) [7] that is multihop, where a member vehicle can connect another neighboring vehicle. This scheme is good for scalability, but there is no localized processing of data. Mobile Gateway Selection Algorithm (MGSA) [8] is good for gateway selection that is based on closeness to the base station. This scheme is fully dependent on the third-party network, so cost incurred and stability is not the priority.

A scheme of clustering that is based on cooperation among vehicles is presented in [5] that is dependent on GPS. Performance is a lack, in case of GPS signal interruptions. Another LTE-dependent clustering scheme is presented in [4] that also relies on the LTE network for location-based information required by the vehicles while traveling on the road, whenever the need to make driving-related decisions. There is no localized mechanism that helps vehicles in the absence of GPS signals. A Fermi learning model based on the prospect theory is developed in [19], which calculates the density of distribution and the distance between the taxis. $\mathrm{Hu}$ et al. [20] proposed an optimal beacon transmission rate allocation for cluster control. An adoptive rate is defined for efficient intracluster communication that is useful in avoiding cluster congestion.

In the absence of GPS, for the vehicles, finding out their position is difficult, which creates a hurdle in communication within the cluster. A geometry-based scheme [21] is introduced that is a cooperative GPS-assisted localization mechanism that works on a mathematical calculation of location. There are other localization schemes [22] those work with GPS and graph theory to know their position on the road. The global navigation satellite system (GNSS) [23] enables precise timing information that is the primary means for vehicle positioning and velocity determination in VANETs. There is one localization scheme [24] that makes use of the roadside unit to increase the accuracy of the GPS in calculating the vehicle's position in VANET, but this is costly to deploy several roadside units.

Route planning and driving assistance applications require heterogeneous network infrastructure that can efficiently and reliably communicate. This necessitates the development of a stable clustering scheme specially designed for TIS because general clustering techniques cannot work well with all types of use cases and network scenarios. In a scenario, where vehicles that depend on GPS perform poorly in urban areas, at the same time, remote servers required smooth transmission of data from vehicles. An efficient scheme is required to be developed for such a scenario.

\section{The Methodology of Self- Localization Scheme}

In Figure 1, a typical urban VANET scenario is depicted. At remote servers, a deep learning-based caching method [25] is an excellent choice to process road traffic data and to predict route planning alters/suggestions. These remote servers gather road traffic data from the vehicles via the Internet by using underlying telecommunication networks. After data processing, alters/suggestions are sent back to vehicles for better driving. For the remote servers to work well, road traffic data exchange should be done smoothly.

The successful exchange of status information among vehicles in the cluster prolongs the CM and cluster life. In urban topography, GPS signals are weakened or disappear because of reflection and refraction due to high-rise buildings and structures (as depicted by Figure 1).

In this situation, all vehicles within the cluster do not receive GPS signals. Thus, their locations cannot be calculated and sent back to $\mathrm{CH}$ and subsequently to the remote servers. Accordingly, SLCA will be initiated by the CMs to calculate the position by utilizing $\mathrm{CH}$ location updates. In our proposed methodology, a cluster is first formed by using criteria (direction, location, average speed, destination, and by keeping in view only interesting vehicles). A modified version of stable interest-aware clustering (SIAC) [4] that included an additional parameter of destination named as destination and interest-aware clustering (DIAC) is proposed. After the formation of the cluster whenever the GPS signal is lost, then CM will not be able to calculate location coordinates and then SLCA is triggered to compute the coordinates as shown in the block diagram of the clustering process in Figure 2.

SLCA mechanism uses the relative change in the velocity of vehicles (i.e., SV and CM) as described in Figure 3. No change in velocity will initiate the arithmetic formulation which adds the initial difference in the coordinates of the $\mathrm{CH}$ and those of the CM to the current coordinates. Otherwise, first, check whether the change in the velocity of the $\mathrm{CH}$ is greater than the change in the velocity of $\mathrm{CM}$. If this change is not greater than the difference in degrees of the initial coordinates of $\mathrm{CH}, \mathrm{CM}$ is subtracted from the current coordinates of the CM. If the change in velocity of the $\mathrm{CH}$ is greater than the difference in degrees of the initial coordinates of $\mathrm{CH}, \mathrm{CM}$ is added to the current coordinates of the $\mathrm{CM}$. This process provides members with their coordinates.

In this way, CMs update their location coordinates and propagate to other vehicles while traveling on the road. SLCA helps CMs within the cluster to maintain a stable connection with $\mathrm{CH}$. This decreases the frequency of link failure and also decreases the frequency of activation of the cluster leaving algorithm. The cluster leaving algorithm works to forcibly remove a CM from the cluster which is not sending location updates to $\mathrm{CH}$ within the specified time. By doing so, SLCA enables CM to be there in the cluster for a long time which increases cluster life. In this way, SLCA once integrated within DIAC increases the stability of the cluster.

3.1. Incorporation of Self-Localization Scheme. The CM calculates its location once receives the location coordinates of the $\mathrm{CH}$ and maintains a record of both location coordinates. Whenever GPS signal is interrupted by any means (e.g., due to high-rise buildings), the SLCA will be invoked 


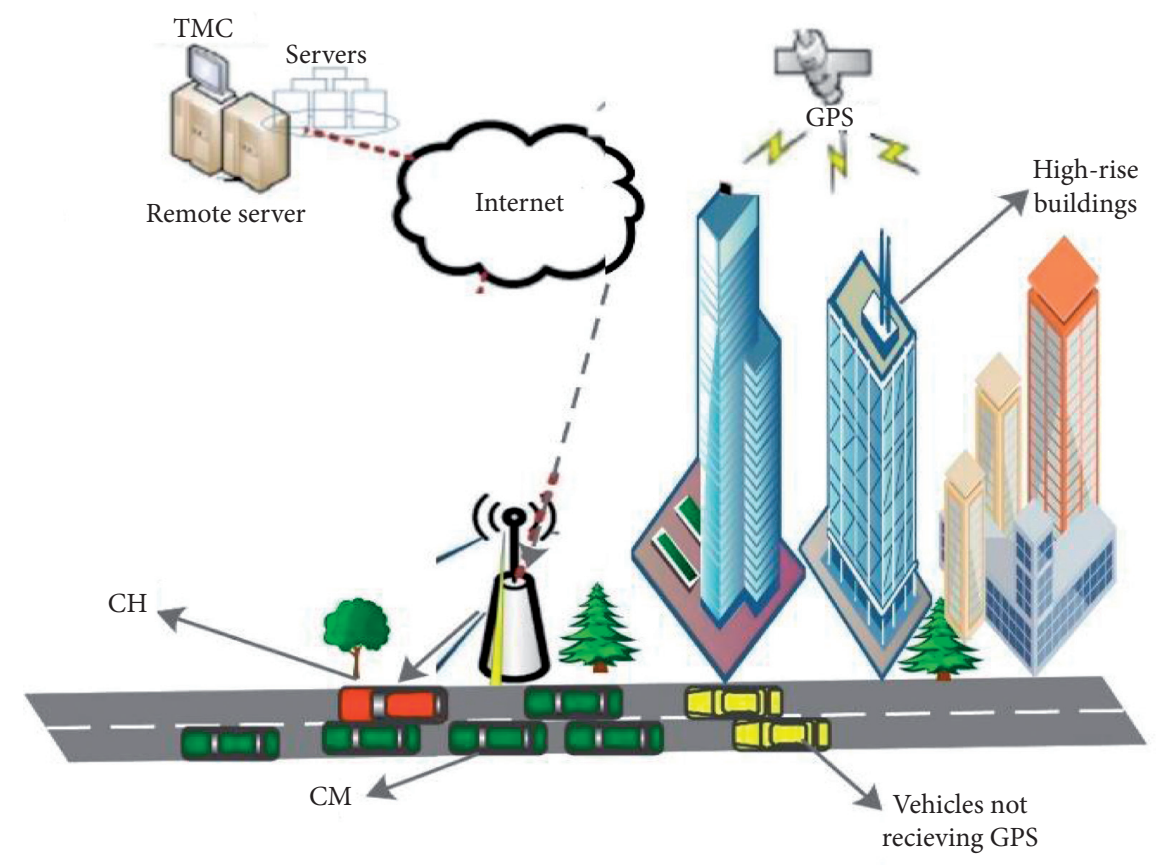

Figure 1: Urbane VANET scenario.

by CM, as described in Algorithm 1. Symbols used in algorithm formulation are defined in Table 1.

The SLCA checks the change in velocity of both $\mathrm{CH}$ and $\mathrm{CM}$ and acts according to Algorithm 1. CM maintains the record related to speed and initial coordinates and calculates differences all the time. Once the cluster is broken apart, all records are reset to zero.

We notice that there is some extra processing that is required for our algorithm to work and the processing time is neglectable.

\section{Implementation}

The additional tools we incorporated into our simulation environment to create a heterogeneous network are Veins [26] and SimuLTE [27]. The heterogeneous vehicular network was created to implement the proposed clustering scheme and SLCA.

4.1. Simulation Setup. The hybrid architecture is built by integrating VANET and LTE to get reliable simulation results. The simulation is repeated for various velocities of vehicles ranging from $10 \mathrm{~m} / \mathrm{s}$ to $35 \mathrm{~m} / \mathrm{s}$. To increase the confidence level, simulation is repeated 3 times at each velocity of vehicles, and the average is taken for comparative analysis in a graphical form. The simulation tools are set up as presented in Figure 4, and the simulation parameters are set as presented in Table 2 .

\section{Results and Discussion}

Some obstacles, such as high-rise buildings, are introduced in the simulation. Underpasses with GPS signal interruptions are also simulated. We test by introducing interruption pauses during our simulation deliberately and check the performance of SLCA. The performance of SLCA-based DIAC (DIAC-LA) is compared with simple DIAC, VMaSC, and MGSA in terms of metrics such as CM duration, number of CMs per cluster, CMs that forcefully left per cluster, PDR, and clustering overhead. The comparison results are shown in Figures 5-9. The first three metrics are measured to check the stability of the proposed scheme, and the last two metrics are to measure the network performance.

Figure 5 shows that the CM duration of DIAC-LA is greater than those of all other approaches at various velocities of vehicles. The reason is that SLCA running at CM sides increases the synchronization between the CMs and $\mathrm{CH}$, which reduces the chances of CMs leaving the cluster. The difference between red and green lines in Figure 5 shows the increase in CM duration when SLCA is incorporated within DIAC. The increase in CM duration shows that the $\mathrm{CMs}$ will remain connected to the $\mathrm{CH}$ for a longer period that results in a longer cluster lifetime. This increase in a cluster lifetime is a clear sign of cluster stability. At the same time, this also makes a smooth exchange of traffic-related data such as the location of every vehicle on to the server.

Figure 6 shows the average number of CMs per cluster at various pause times of simulation. The number of CMs per cluster for VMaSC and MGSA is higher than those for DIAC and DIAC-LA. The reason is that VMaSC allows multihop clustering and has different clustering criteria. However, the number of CMs per cluster for DIAC-LA is higher than that for simple DIAC, which shows an increase in the number of $\mathrm{CMs}$ within the cluster. At various pause times, there is no significant change in the number of CMs as compared to the DIAC. This also shows stability within cluster over time as there are no many CM layoffs. At various pause times, a large 


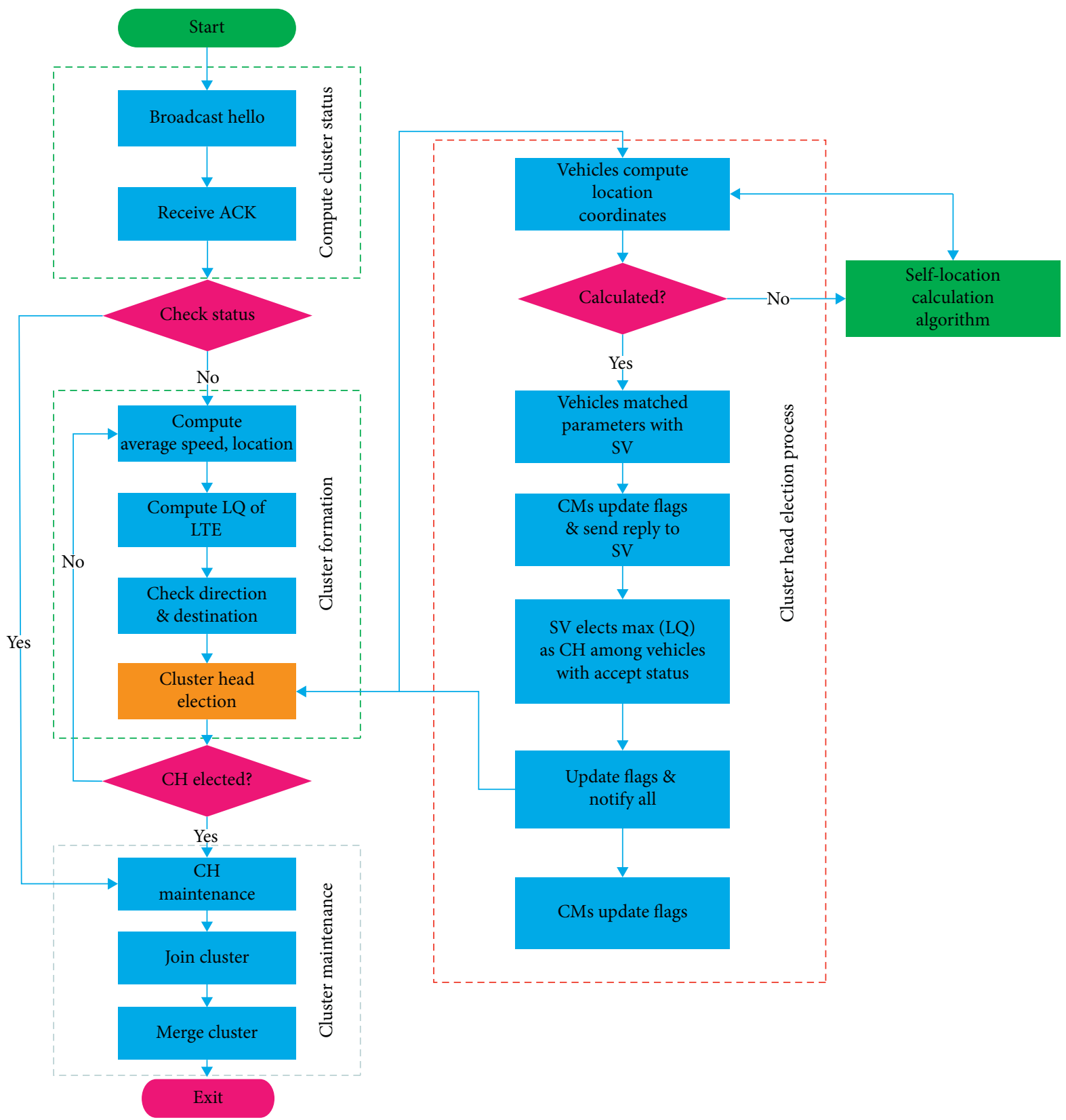

FIgURE 2: Block diagram of clustering process.

number of CMs remain with $\mathrm{CH}$ in DIAC-LA. Therefore, the incorporation of SLCA provides sustainability to CMs within the cluster.

Figure 7 shows the percentage of vehicles forced to leave per cluster at various velocities of vehicles. The percentage for DIAC-LA is lower than that for DIAC. The percentage of force leaving of CMs for DIAC-LA and DIAC is increasing with the increase in velocity of the vehicles. However, the percentage of DIAC-LA remains lower than DIAC at different velocities throughout the simulation. The reason is that, when CMs can calculate their locations in the absence of GPS signals, their synchronization with $\mathrm{CH}$ increases, and the frequency of $\mathrm{CHs}$ applying forced leaving procedure to $\mathrm{CMs}$ decreases. When $\mathrm{CH}$ is not hearing from a $\mathrm{CM}$ for a period, it forcefully removes that CM from the cluster. In our case, SLCA allows CMs to calculate their location and coordinate with $\mathrm{CH}$ for a long period even in the absence of GPS signals. The forced leaving increases with the increase in the speed of vehicles, but in the start, when the vehicle velocity is 5 , DIAC shows a better percentage than DIACLA. There is no logical reason to say, maybe at the very beginning, the clustering of DIAC behaves more stable. However, DIAC-LA is still more synchronized than DIAC.

To check the general network performance, the PDR of both DIAC-LA and DIAC is calculated. The PDR of DIACLA is greater than that of simple DIAC, and it remains higher at various velocities of vehicles, as shown in Figure 8. DIAC-LA has a long connection with $\mathrm{CH}$ than DIAC, which 


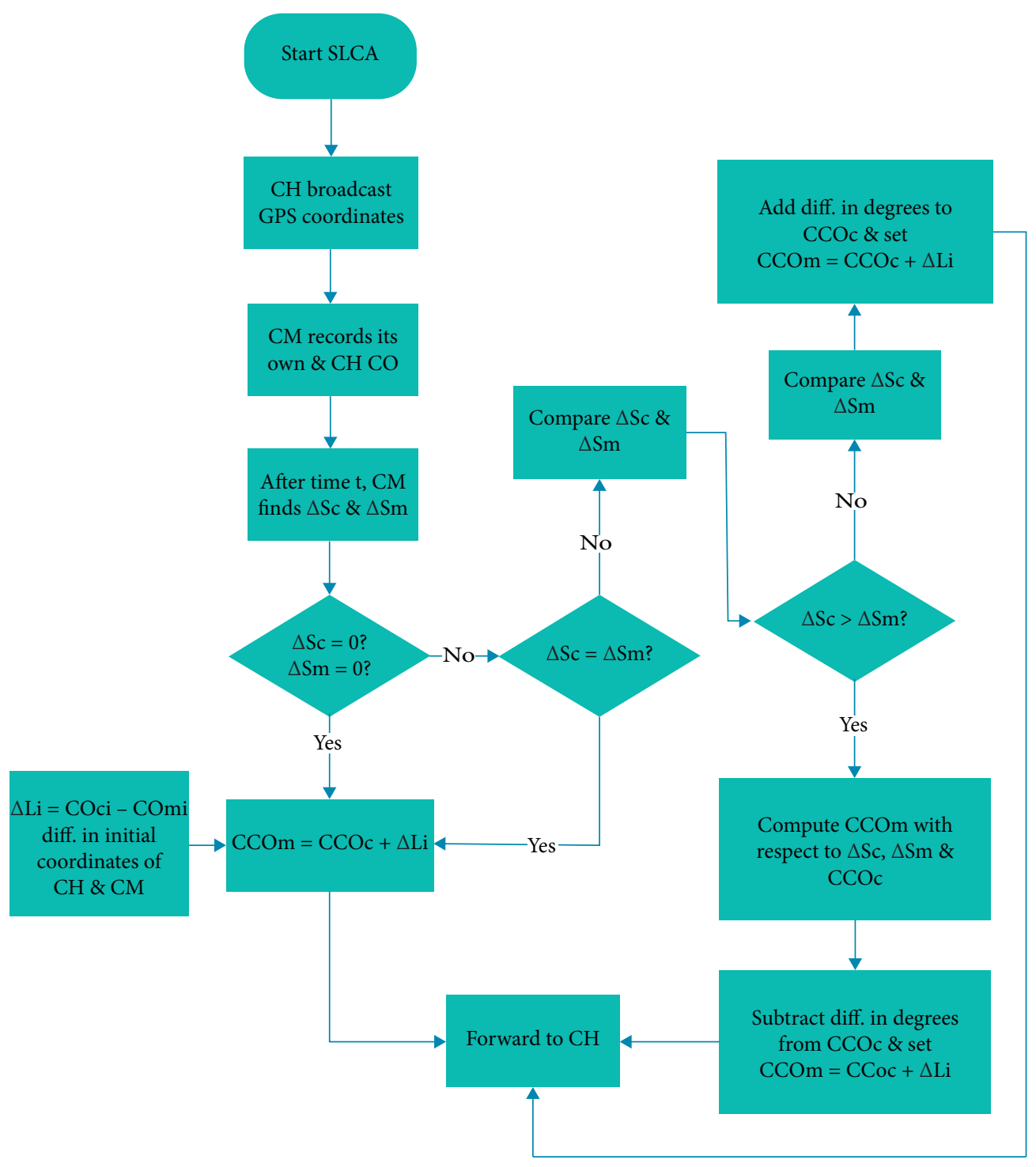

FIgURE 3: Flow diagram of self-location calculation mechanism.
(1) GPS coordinates are propagated by $\mathrm{CH}$
(2) Records ( $\mathrm{CCOm}$ and $\mathrm{CCOc}$ ) maintained by $\mathrm{CM}$
(3) At time $t$, CM loss GPS computes $\Delta \mathrm{Sc} \& \Delta \mathrm{Sm}$
(4) IF $(\Delta \mathrm{Sc}=\Delta \mathrm{Sm})$ AND OR $(\Delta \mathrm{Sc}=0$ AND $\Delta \mathrm{Sm}=0)$
(5) FIND $\Delta \mathrm{Li}=\mathrm{COci}-\mathrm{COmi}$
(6) $\mathrm{FIND} \mathrm{CCOm}=\mathrm{CCO}+\Delta \mathrm{Li}$
(7) ELSE
(8) $\quad$ IF $(\Delta \mathrm{Sc}>\Delta \mathrm{Sm})$
(9) Calculate $\mathrm{CCOm}, \Delta \mathrm{Sc}, \Delta \mathrm{Sm}$, and $\mathrm{CCOc}$
(10) Calculate $\mathrm{CCOm}=\mathrm{CCO}-\Delta \mathrm{li}$
(11) ELSE Calculate $\mathrm{CCOm}=\mathrm{CCOc}+\Delta \mathrm{Li}$
(12) $\mathrm{CM}$ propagates to $\mathrm{CH}$
(13) END

Algorithm 1: Self-location calculation process. 
TABle 1: Symbol definitions.

\begin{tabular}{lc}
\hline Symbol & Description \\
\hline$t$ & Time \\
$\mathrm{CCOc}$ & $\mathrm{CH}$ coordinates after time $t$ \\
$\mathrm{CCO}_{\mathrm{m}}$ & $\mathrm{CM}$ coordinates after time $t$ \\
$\mathrm{COc}_{\mathrm{i}}$ & Initial coordinates of $\mathrm{CH}$ \\
$\mathrm{COm}$ & Initial coordinates of $\mathrm{CM}$ \\
$\Delta \mathrm{Li}$ & The initial change in coordinates of $\mathrm{CH}$ and $\mathrm{CM}$ \\
$\Delta \mathrm{Sc}$ & Change in the speed of $\mathrm{CH}$ \\
$\Delta \mathrm{Sm}$ & Change in the speed of $\mathrm{CM}$ \\
\hline
\end{tabular}

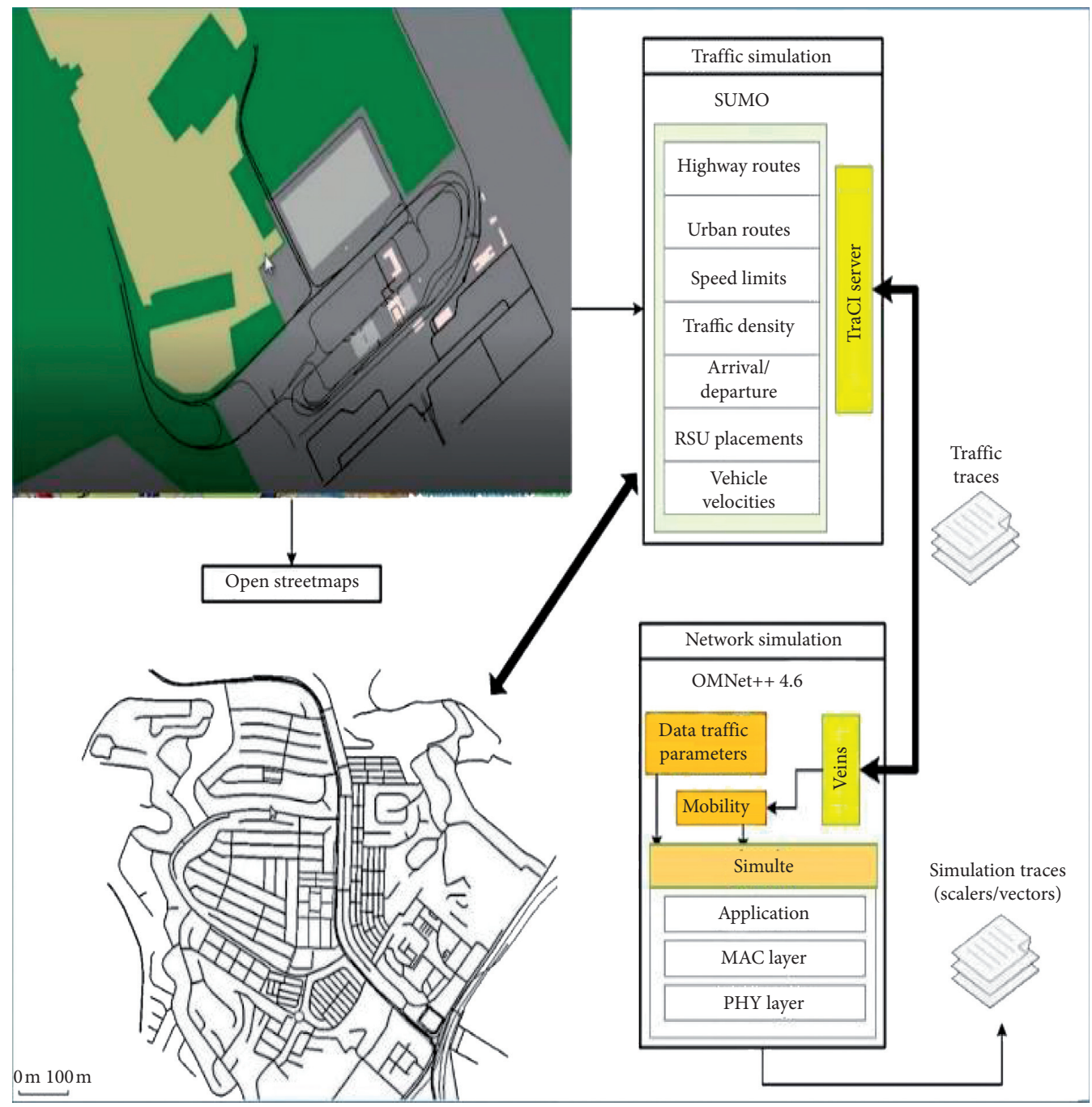

Figure 4: Simulation and evaluation setup.

is the reason for the higher PDR of the latter than the former. At velocities of vehicles of 5 and 10, PDR remains the same for both mechanisms and is high for DIAC-LA under the rest of the velocities. However, overall, the PDR is decreasing with the increase in velocities of the vehicles, but the PDR of DIAC-LA remains low.

To check further network performance, we calculated and compared the clustering overhead of our proposed DAIC-LA and other existing approaches. The DIAC-LA has lower values than all other approaches at various velocities of vehicles, as shown in Figure 9. At various GPS failure rates, all approaches show different clustering overhead. MGSA shows the highest value, and DIAC-LA shows the lowest values. At a failure rate of 1 to 3 , there is a minor difference between DAIC and DIAC-LA, but after that, the clustering overhead of DIAC went higher. Overall, the clustering overhead of DIAC shows a stable performance throughout the simulation time. This result is due to the small 
TABLE 2: Simulation parameter setting.

\begin{tabular}{lc}
\hline Parameters & Values \\
\hline Simulation area & $2 \mathrm{~km} \times 2 \mathrm{~km}$ \\
IEEE 802.11p (frequency/BW) & $5.89 \mathrm{GHz} / 10 \mathrm{MHz}$ \\
Data rate & $5 \mathrm{Mbps}$ \\
Beacon interval & $20 \mathrm{~ms}$ \\
Maximum velocity & $10-35 \mathrm{~m} / \mathrm{s}$ \\
Base station height & $15 \mathrm{~m}$ \\
Control subcarriers & 500 \\
No. of subcarriers per RB & 12 \\
LTE vehicles antenna type & Omnidirectional $(8 \mathrm{dBi} / 6 \mathrm{dBi})$ \\
Simulation time & $300 \mathrm{~s}$ \\
RSU antenna type & Directional \\
OBU vehicle antenna type & Omnidirectional \\
OBU height & $1.5 \mathrm{~m}$ \\
UE TxPower & $20 \mathrm{dBm}$ \\
LTE BS antenna type & $3-\mathrm{sector} \mathrm{directional}$ \\
Vehicle height & $1.5 \mathrm{~m}$ \\
ENodeB TxPower & $45 \mathrm{dBm}$ \\
Number of vehicles & 100 \\
Resource blocks & 100 \\
Guard subcarriers & 423 \\
\hline
\end{tabular}

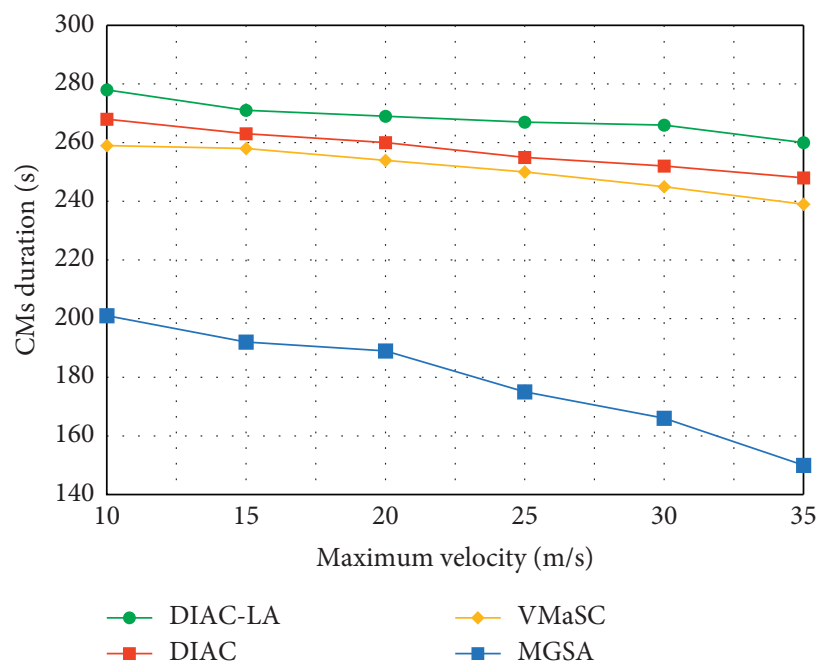

FIgure 5: Comparison of DIAC with and without SLCA.

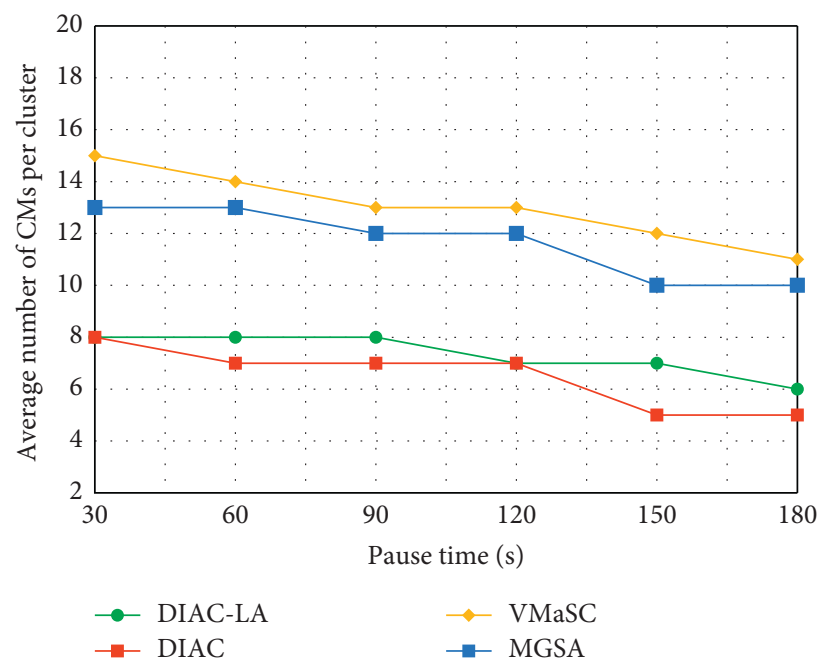

Figure 6: Number of CMs per cluster at different pause times of simulation. 


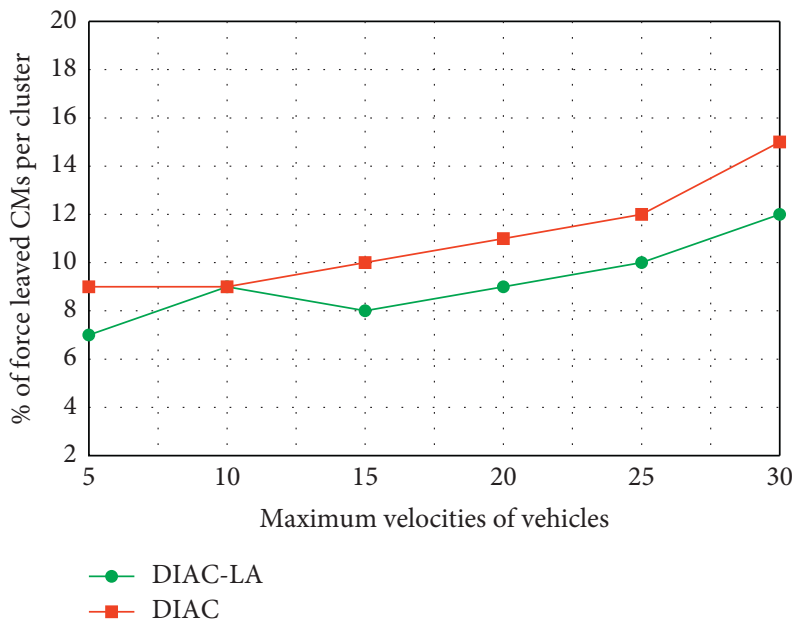

Figure 7: Percentage of vehicles that forcefully leave per cluster at various velocities of vehicles.

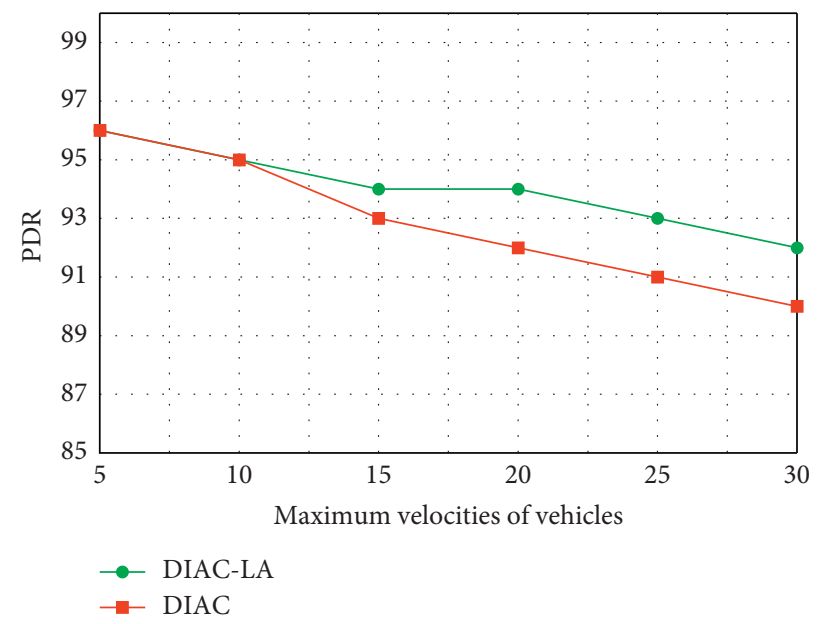

FIgURE 8: The difference in PDR at maximum velocities of vehicles.

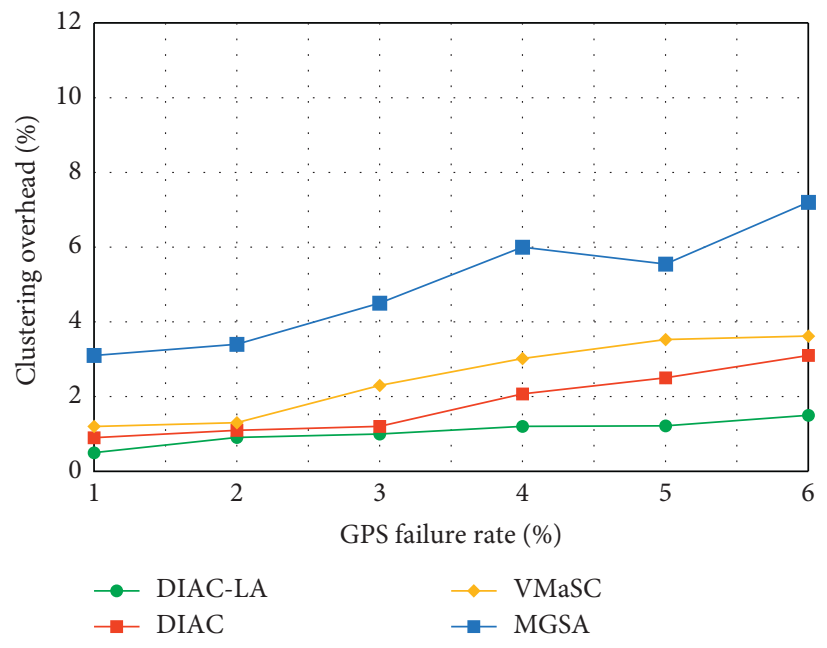

FIGURE 9: Clustering overhead against a preset GPS failure rate. 
fluctuation of CMs in the cluster, which causes stability and few times of reclustering. However, there is a bit decrease in the clustering overhead of MGSA at GPS failure rate from 4 to 5 , but it is still much higher than all other approaches, so this fluctuation is negligible.

In all performance comparisons, DIAC-LA shows better results than VMaSC, MGSA, and especially DIAC. These improved results validate the increase in the synchronization of CMs with $\mathrm{CH}$, which increases the overall cluster life and stability. The increase in performance of the DAIC-LA not only gives stability to the vehicles but also aids the server by smoothening the exchange of data between the vehicles and server.

\section{Conclusion}

The introduction of SLCA within DIAC extends cluster life which shows the stability of the cluster. More stability means greater performance as we observed in our results that the PDR increases and clustering overhead decreases. The SLCA incorporation in the SIAC clustering mechanism improves the vehicular network capabilities which enable the IoV to perform better under complex (heterogeneous network) network scenarios. The localization of data processing at vehicles has a positive impact on the third-party network (entities) such as telecommunication networks and remote servers.

\section{Data Availability}

The data used to support the findings of this study are included in the article.

\section{Conflicts of Interest}

The authors declare that they have no conflicts of interest.

\section{Acknowledgments}

The authors are grateful to the Taif University Researchers Supporting Project number (TURSP-2020/36), Taif University, Taif, Saudi Arabia. The authors are also grateful to the Deanship of Scientific Research, King Saud University for funding this research project through Vice Deanship of Scientific Research Chairs (DSRVCH). This research work was also partially supported by the Faculty of Computer Science and Information Technology, University of Malaya under Postgraduate Research Grant (PG035-2016A).

\section{References}

[1] M. Alam, J. Ferreira, and J. Fonseca, "Introduction to intelligent transportation systems," in Intelligent Transportation Systems: Dependable Vehicular Communications for Improved Road Safety, pp. 1-17, Springer International Publishing, NY, USA, 2016.

[2] W. Wang, F. Xia, H. Nie et al., "Vehicle trajectory clustering based on dynamic representation learning of internet of vehicles," IEEE Transactions on Intelligent Transportation Systems, pp. 1-10, 2020.
[3] D. Zhang, H. Ge, T. Zhang, Y.-Y. Cui, X. Liu, and G. Mao, "New multi-hop clustering algorithm for vehicular ad hoc networks," IEEE Transactions on Intelligent Transportation Systems, vol. 20, no. 4, pp. 1517-1530, 2019.

[4] I. Ahmad, R. Md Noor, and M. Reza Z'aba, "LTE efficiency when used in traffic information systems: a stable interest aware clustering," International Journal of Communication Systems, vol. 32, no. 2, p. e3853, 2019.

[5] I. Ahmad, R. M. Noor, M. R. Zaba, M. A. Qureshi, M. Imran, and M. Shoaib, "A cooperative heterogeneous vehicular clustering mechanism for road traffic management," International Journal of Parallel Programming, vol. 48, no. 5, pp. 870-889, 2020.

[6] O. Briante, C. Campolo, A. Iera, A. Molinaro, S. Y. Paratore, and G. Ruggeri, "Supporting augmented floating car data through smartphone-based crowd-sensing," Vehicular Communications, vol. 1, no. 4, pp. 181-196, 2014.

[7] S. Ucar, S. C. Ergen, and O. Ozkasap, "Multihop-cluster-based IEEE 802.11p and LTE hybrid architecture for VANET safety message dissemination," IEEE Transactions on Vehicular Technology, vol. 65, no. 4, pp. 2621-2636, 2016.

[8] A. Benslimane, T. Taleb, and R. Sivaraj, "Dynamic clusteringbased adaptive mobile gateway management in integrated VANET - 3 G heterogeneous wireless networks," IEEE Journal on Selected Areas in Communications, vol. 29, no. 3, pp. 559-570, 2011.

[9] F. Hagenauer, F. Dressler, and C. Sommer, "Poster: a simulator for heterogeneous vehicular networks," in Proceedings of the Vehicular Networking Conference (VNC), 2014, IEEE, Paderborn, Germany, December 2014.

[10] D. Krajzewicz, J. Erdmann, M. Behrisch, and L. Bieker-Walz, "Recent development and applications of SUMO-Simulation of Urban MObility," International Journal on Advances in Systems and Measurements, vol. 5, no. 3\&4, pp. 128-138, 2012.

[11] R. Alroobaea, "An empirical combination of machine learning models to enhance author profiling performance," International Journal of Advanced Trends in Computer Science and Engineering, vol. 9, no. 2, pp. 2130-2137, 2020.

[12] Z. H. Mir and F. Filali, "LTE and IEEE $802.11 \mathrm{p}$ for vehicular networking: a performance evaluation," EURASIP Journal on Wireless Communications and Networking, vol. 2014, no. 1, p. 1, 2014.

[13] K. Zheng, Q. Zheng, P. Chatzimisios, W. Xiang, and Y. Zhou, "Heterogeneous vehicular networking: a survey on architecture, challenges, and solutions," IEEE Communications Surveys \& Tutorials, vol. 17, no. 4, pp. 2377-2396, 2015.

[14] M. Gerla, C. Wu, G. Pau, and X. Zhu, "Content distribution in VANETs," Vehicular Communications, vol. 1, no. 1, pp. 3-12, 2014.

[15] C. Wu, M. Gerla, and N. Mastronarde, "Incentive driven LTE content distribution in VANETs," in Proceedings of the 2015 14th Annual Mediterranean Ad Hoc Networking Workshop (MED-HOC-NET), June 2015.

[16] J. Wang, "Dynamic clustering and cooperative scheduling for vehicle-to-vehicle communication in bidirectional road scenarios," IEEE Transactions on Intelligent Transportation Systems, vol. 19, no. 6, 2017.

[17] C. Lottermann, M. Botsov, P. Fertl et al., "LTE for vehicular communications," in Vehicular Ad Hoc Networks, pp. 457-501, Springer, NY, USA, 2015.

[18] K. K. Rana, S. Tripathi, and R. S. Raw, "Analytical analysis of improved directional location added routing protocol for VANETS," Wireless Personal Communications, vol. 98, no. 2, pp. 2403-2426, 2018. 
[19] W. Zhang and Y. Fan, "Spatiotemporal characteristics and self-organization of urban taxi dispatch," Complexity, vol. 2020, Article ID 3659315, , 2020.

[20] L. Hu, Y. Xiao, and Z. Dai, "Beacon transmission rate allocation optimization under synchronized P-persistent repetition MAC protocol for platooning," Wireless Communications and Mobile Computing, vol. 2020, Article ID 8887134, 2020.

[21] O. Kaiwartya, Y. Cao, J. Lloret et al., "Geometry-based localization for GPS outage in vehicular cyber physical systems," IEEE Transactions on Vehicular Technology, vol. 67, no. 5, pp. 3800-3812, 2018.

[22] A. Sobehy, E. Renault, and P. Muhlethaler, "Position certainty propagation: a location service for MANETs," in Proceedings of the MSPN 2018-4th International Conference on Mobile, Secure, and Programmable Networking, pp. 131-142, Springer, Paris, France, June 2018.

[23] K. F. Hasan, Y. Feng, and Y.-C. Tian, "GNSS time synchronization in vehicular ad-hoc networks: benefits and feasibility," IEEE Transactions on Intelligent Transportation Systems, vol. 19, no. 12, pp. 3915-3924, 2018.

[24] F. A. Santos, A. T. Akabane, R. S. Yokoyama, A. A. F. Loureiro, and L. A. Villas, "A roadside unit-based localization scheme to improve positioning for vehicular networks," in Proceedings of the 2016 IEEE 84th Vehicular Technology Conference (VTC-Fall), Montreal, Québec City (QC), Canada, September 2016.

[25] A. Ndikumana, N. H. Tran, D. H. Kim, K. T. Kim, and C. S. Hong, "Deep learning based caching for self-driving cars in multi-access edge computing," IEEE Transactions on Intelligent Transportation Systems, pp. 1-16, 2020.

[26] C. Sommer, Veins: Vehicles in Network Simulation, 2015, https://veins.car2x.org/.

[27] A. Virdis, G. Stea, and G. Nardini, "Simulating LTE/LTEadvanced networks with SimuLTE," Advances in Intelligent Systems and Computing, vol. 402, pp. 83-105, 2015. 